\title{
INGENIERÍA INDUSTRIAL
}

\section{¿Por qué los videojuegos son una alterna- tiva de negocio en la ciudad de Bogotá?}

Why videogames are a business alternative in

Bogotá?

Camilo Estacio ${ }^{1}$

Artículo de investigación

Recepción: 29 de abril de 2020 Aceptación: 09 de junio de 2020

Cómo citar:

Estacio, C. 2020. ¿Por qué los videojuegos son una alternativa de negocio en la ciudad de Bogotá? Mare Ingenii. Ingenierías 2 (1), pp. 47-54. Recuperado de http://cipres.sanmateo.edu.co/index.php/mi

1 Docente en la Corporación Universitaria Unigermana: Correo electrónico: docente.camilo.estacio@unigermana.edu.co 


\section{Resumen:}

La industria de los videojuegos crecido mucho en los últimos años. Ha recaudado ganancias millonarias, ha llegado al punto de crear economías propias, elevarse a nivel de arte, y tener millones de aficionados y seguidores alrededor del mundo. Latinoamérica es un poderoso mercado emergente, donde hay cada vez más personas interesadas en el negocio. La investigación presentada pretende dar un panorama sobre cómo se mueve la industria en Bogotá, ya que buena parte se desarrolla en la capital del país. La investigación aborda el tema a partir de tres (3) ejes principales: la venta y comercialización de videojuegos, la creación, diseño y programación de juegos y la gestión de esports. Para ello, se recurre a datos proporcionados por especialistas y entrevistas a personas involucradas en la industria de los videojuegos. De esta manera, el lector puede tener un acercamiento al panorama general de la industria para Bogotá.

Palabras claves: videojuegos; esports; competitividad.

\section{Abstract:}

The video game industry has grown with incredible speed in recent years. From the creation of Pong until the last release of the newest video game, the industry has raised a lot of profits, it has reached the goal of creating own economies, rising to the level of art, and having millions of fans and followers around the world. For large companies dedicated to video games, Latin America is a powerful emerging market, where more and more people interested in the business can be found. This research wants to know first-hand how the industry moves in Bogotá, since much of it develops there.

The research approaches this topic from three (3) main axes: the sale and commercialization of video games, the creation, design and pro- gramming of games and esports management. This way, it will be possible to have an approach to the general panorama of the industry for Bogotá.

Keywords: Video games; eSports; competitiveness.

\section{Introducción}

La ola de la economía naranja iniciada en el gobierno Santos (y potenciada en el Gobierno actual) crean una coyuntura favorable para el desarrollo de la industria. Sin embargo, se está hablando de un negocio en el que otros países de Latinoamérica ya llevan mucha ventaja, como pasa con Chile, Brasil y especialmente México. Existen otros países como Costa Rica, que ven a Colombia como competencia directa y la han analizado a fin de mejorar su propia propuesta de valor.

Lamentablemente, son pocas las instituciones que dedican esfuerzos al estudio y el mejoramiento de la industria dentro del país, pero aun así se han presentado interesantes logros al respecto. Tales instituciones y personas no están interesadas en hacer negocio (al menos no principalmente), sino personas que se autodenominan fanáticos, lo que da una de las características más especiales de la industria: es un negocio de fans, para fans.

Los hallazgos que hasta el momento se han dado reflejan las principales oportunidades y amenazas que tiene Colombia como potencial proveedor de productos y servicios enfocados a videojuegos, y han dejado ver algunas de las fortalezas y debilidades del sector en Colombia. Así, deben comenzar a corregirse ahora, si se desea 
que Colombia aproveche el crecimiento que la industria está presentando a nivel mundial.

\section{Objetivos.}

El objetivo general consiste en analizar el nivel de competitividad de la industria de los videojuegos en Bogotá, a partir de un reconocimiento del estado del arte, debilidades, fortalezas y necesidades. Por otro lado, los objetivos específicos son tres (3), a continuación:

- Especificar el estado actual de la industria de los videojuegos en Colombia, con el fin de tener claridad sobre los diferentes subsectores donde ésta se mueve en el país.

- Ilustrar las fortalezas y debilidades generales en cada uno de los subsectores hallados para la cuidad de Bogotá, con el fin de tener un panorama general de la situación del sector a nivel competitivo.

- Detallar las coyunturas actuales de la industria y del país que intervienen positiva o negativamente en su desarrollo, para así tener un cuadro de competitividad a nivel nacional.

\section{Marco teórico}

Como se especificó con anterioridad, la investigación tiene en cuenta tres (3) ejes, a saber:

\section{Compraventa de videojuegos.}

Este eje hace referencia a los diferentes comercios que se dedican de forma exclusiva o no exclusiva, a la compra y venta por mayor valor, de videojuegos, consolas, accesorios $y$, en general, hardware y sof- tware dedicado para videojuegos, ya sea en consolas de mesa, portátiles, computadores, etc.

\section{Diseño y programación de vi- deojuegos.}

Este eje se refiere a aquellas empresas dedicadas a crear diseños o programar desde partes específicas de un videojuego (personajes, escenarios, mecánicas, etc.), hasta juegos enteros, ya sea para distribución por propia mano o a través de Publisher.

\section{Gestión de eSports.}

Este eje trata de empresas dedicadas a los esports de diferentes formas, como lo son la organización de torneos, la gestión y el patrocinio de equipos o competidores individuales de videojuegos a nivel competitivo.

\section{La compraventa de videojue- gos.}

Respecto al primer eje, el negocio de compraventa de videojuegos tiene varios actores:

- Las grandes superficies. Son las cadenas de retail que venden todo tipo de productos, e incluyen a los videojuegos dentro de su línea tecnológica. Los ejemplos más claros son las cadenas Fallabella, K-Tronix y Panamericana.

- Los pequeños negocios: Son comercios pequeños que se dedican de forma casi exclusiva a la compraventa de videojuegos. Se trata de tiendas especializadas que pueden funcionar de diversas formas, como son tiendas físicas, tiendas online (con entrega a domicilio), y negocios organizados de juegos usados. 
- Los negocios en internet: Estos negocios son ofertados por grandes empresas tecnológicas, generalmente dedicadas a la venta de juegos a través de internet, por medio de descargas, como son los servicios en nube de PlayStation Network, Xbox Game Pass, Google Stadia, Apple Arcade y Steam, entre otros.
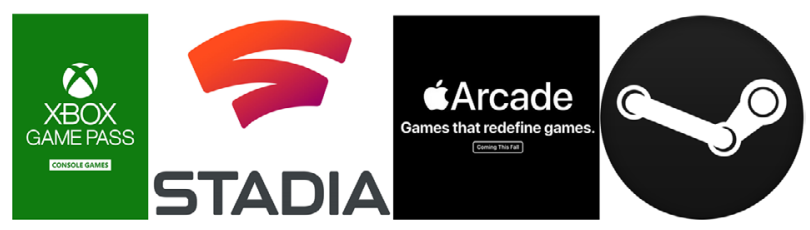

Figura 1. Algunas plataformas de juego en línea.

Estos juegos suelen promocionarse a través de internet, con el apoyo de canales de gamers, streamers y personalidades con presencia activa en plataformas como Facebook, Twitter, Twitch y Youtube, además de los canales y estrategias tradicionales.

\section{El desarrollo de videojuegos.}

Los videojuegos sufren de procesos cíclicos para su creación que pasan por la creación de la idea, una fase de diseño de la cual sale un prototipo, la creación de una versión beta, y un control de calidad. A partir de los resultados del control de calidad, el ciclo vuelve a comenzar y evoluciona de esta forma a través de las macro fases de producción, mostradas en la ilustración a continuación [1].

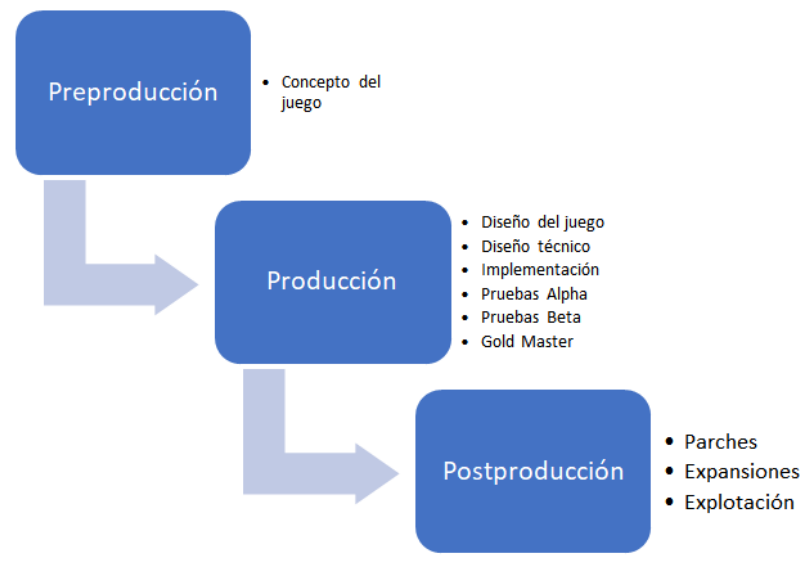

Figura 2. Etapas de creación de un videojuego.

Entonces, dependiendo de la escala del videojuego, en cada una de las etapas de producción del videojuego puede intervenir una o varias empresas. Los videojuegos más grandes echan mano de varias compañías que se encargan de diferentes partes de este, al punto de emplear empresas contratistas al mismo nivel de la industria cinematográfica. Se requieren expertos en programación, diseño, musicalización, fotografía, calidad, comercialización, publicidad, traducción, etc.

\section{Auge y actualidad de los es- ports.}

En la actualidad, los eSports son uno de los rubros más rentables de toda la industria. Según Forbes [2] los principales equipos de eSports tienen valores entre US50 millones y US210 millones, con ganancias entre US4 millones y US22 millones al año. Los juegos más representativos para estos equipos son League of Legends, Fortnite, Counter Strike, DOTA2, Overwatch, Rainbow Six Siege, FIFA, Super Smash Bros y Clash Royale, pero no son los únicos, pues existen muchos juegos en línea que pueden funcionar como eSports. La aceptación a nivel general de los videojuegos es innegable, no solamente por los números presentados, sino por otras investigaciones, como las desarrolladas por el Banco 
Interamericano de Desarrollo (BID) [3], la Cámara de Comercio de Bogotá [4], [5], e investigadores como Alonqueo y Rehbein en Chile [6], Mariglia Danielli e Isabel Dueñas en Perú [7], Alejandro Álvarez y Julian Barrera en Colombia [8], entre otros.

El poder económico de los esports da para mover anualmente más de US1500 millones. Se prevé que para el año 2022, los esports produzcan cerca de US2.300 millones entre torneos, patrocinios, publicidad, boletería, derechos de transmisión, etc. [9]. Actualmente, marcas como RedBull, BMW, los clubes deportivos Shalke04 de Alemania, Chelsea de Inglaterra, Fútbol Club Valencia de España, la Universidad Católica de Chile, el Clube de Regatas do Flamengo (Brasil) y marcas como Hewlett Packard, Gillette, Sketchers, entre muchas otras, patrocinan, organizan o poseen torneos o equipos de esports.

El tema de los videojuegos es importante a nivel de negocios; movió alrededor de US137 billones en 2018 y ha tenido crecimientos anuales alrededor del 10\% anual desde 2012 [9]. De ese total, menos del 5\% pasa por Latinoamérica, mientras que cerca del 50\% se queda en Asia - Pacífico, 23\% en Norteamérica y 21\% en Europa y medio Este. Específicamente en Latinoamérica, Colombia ocupa el cuarto lugar en cuanto a ingresos generados, detrás de México, Argentina y Brasil, y seguido de cerca por Chile.

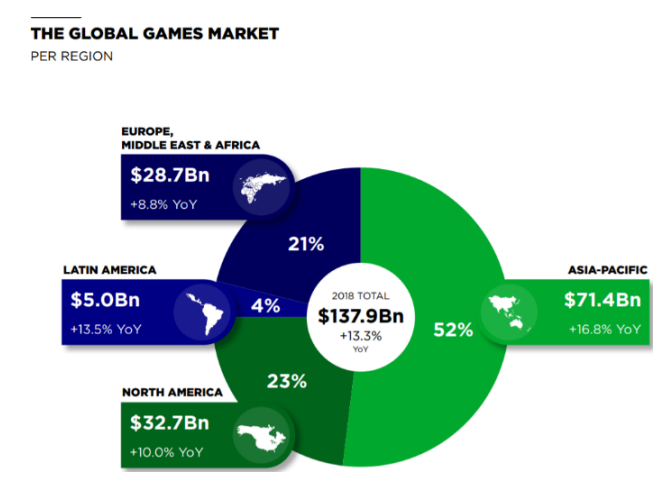

Figura 3.Mercado mundial de videojuegos por región.

\section{Metodología}

La presente investigación presenta un enfoque mixto (análisis cualitativo con elementos de apoyo cuantitativos), un tipo exploratorio con alcance analítico. Además, se usan métodos de tipo deductivo. Las herramientas y técnicas se resumen en la siguiente tabla.

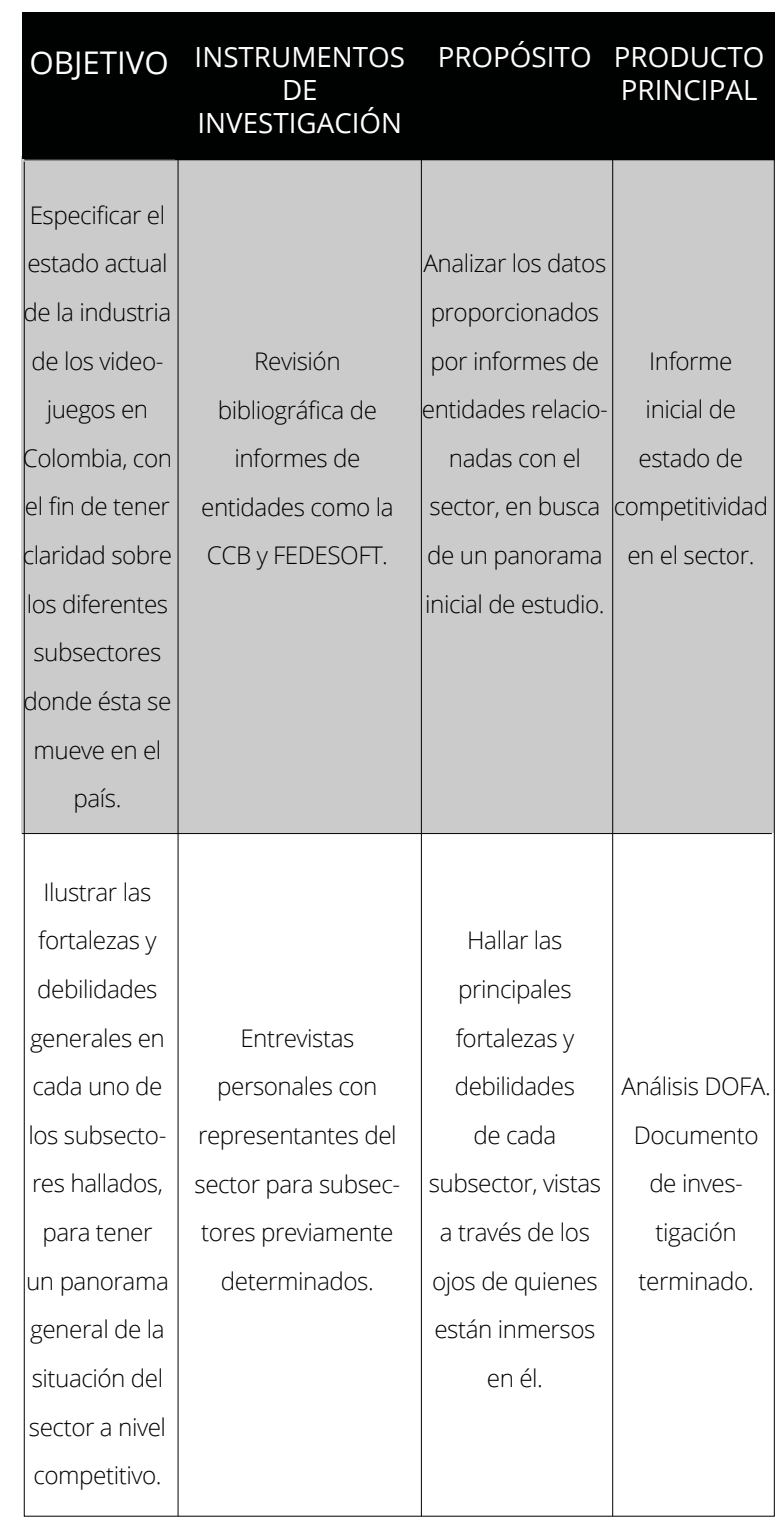




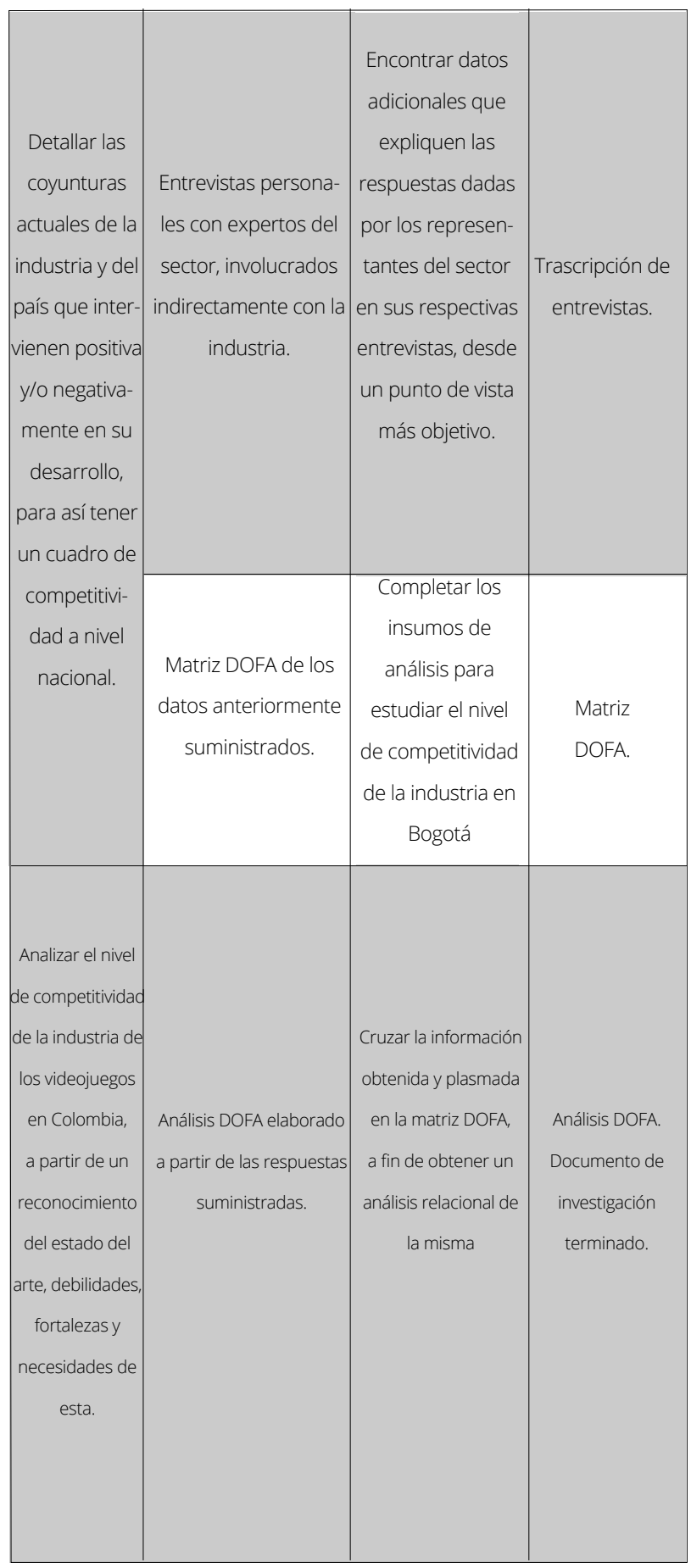

Tabla 1. Herramientas y técnicas usadas en la investigación.

\section{Desarrollo}

Inicialmente, para el desarrollo de esta investigación, se recabó toda la información posible referente al tema de los videojuegos en Colombia, encontrando do- cumentos de la Cámara de Comercio de Bogotá, tesis de grado de diferentes universidades y estudios elaborados por PROCOLOMBIA, entre otros. A partir de ellos se lograron ver las primeras debilidades del sector, como lo son la falta de caracterización de las empresas de la industria ante entidades fiscales o tributarias, la información desactualizada de algunos estudios, etc. Por otro lado, también se encontraron fortalezas, como la calidad de la técnica en programadores y diseñadores, la posición geográfica del país, etc.

En la segunda fase de la investigación, se plantearon entrevistas semiestructuradas con actores directos de la industria (programadores y creadores de videojuegos, gestores de torneos de esports, propietarios de tiendas de videojuegos, etc.), con el propósito de contrastar sus opiniones con las encontradas en la parte inicial de la indagación.

En la tercera parte de la investigación, se espera contactar con personas relacionadas con el tema de forma más indirecta, especialmente relacionados con el tema de economía naranja y el sector académico. Lo anterior, con el fin de conocer sus opiniones y saber qué se está haciendo por la industria, a la luz de un análisis DOFA creado con la información obtenida anteriormente.

\section{Resultados}

En lo que va corrido de la investigación, se puede concluir que entre las fortalezas más grandes que tiene Bogotá para incursionar con éxito en la industria de los videojuegos está la gran creatividad de programadores y diseñadores, la pasión por su trabajo, las ayudas que el gobierno ofrece para impulsar economías creativas, el apoyo a la industria de parte de varias organizaciones del gobierno y la alta competitividad en costos respecto a otros paí- 
ses. Las debilidades más grandes son los esfuerzos aislados y la alta competencia interna traducida en egoísmo, y la falta de competencias blandas en los profesionales que se dedican a la industria (especialmente idiomas y habilidades de negociación y venta), sin mencionar temas tributarios sobre los contenidos digitales en el país.

Las fortalezas que tiene Bogotá (y Colombia) para ser fuertes en la industria son la alineación de husos horarios con los clientes más grandes (EE. UU. y Canadá), la cercanía y facilidad de traslado entre EE. UU. y Colombia. Como amenazas está la altísima competencia de países más desarrollados en el tema, como lo son México y Chile, y el interés por incursionar en el mercado por parte de otras economías, como pasa con Costa Rica. Una de las pruebas más importantes de lo anterior es que la base de operaciones de una de las empresas más reconocidas del mundo en cuanto a motor gráfico para videojuegos refiere, montó sus operaciones para Latinoamérica en Colombia [10].

\section{Recomendaciones}

Los hallazgos de esta investigación fueron útiles para tener un panorama general de la industria bogotana de los videojuegos. Sin embargo, muchos detalles se omitieron, o no se profundizaron debidamente. Por lo tanto, las recomendaciones más importantes derivadas de esta investigación son las siguientes:

Las empresas dedicadas al diseño de personajes, la programación de mecánicas $y$, en general, a proveer servicios tercerizados para Publisher (incluida el diseño y programación de juegos enteros) requiere una investigación aparte; constituyen una gran oportunidad para que el país de un importante paso en la migración de su economía, de la producción de commodities a la exportación de productos y servicios digitales, parte fundamental de la creciente economía creativa.

Algo similar ocurre con los esports; toda vez que en Colombia ya existen organizaciones dispuestas a trabajar de forma colaborativa; tienen claro lo que se debe hacer para poner al país en un lugar privilegiado a nivel latinoamericano y, por qué no, mundial. Es necesario que gobierno, empresa privada y academia pongan sus ojos en la industria, sepan ver y aprovechar sus grandes ventajas y apliquen tiempo y recursos en su explotación. Para ello, es necesario estudiar este segmento de la industria como un terreno con sus propias dinámicas.

Por su lado, el segmento de compraventa de videojuegos parece sufrir de los mismos pesares que los productos tipo software y hardware de otro tipo. Este tema tiene la característica de ser muy hermético respecto a la búsqueda de información. Para llevar a cabo un estudio como el que se pretendió en este documento, será necesario recurrir a otras herramientas que no pasen por preguntar directamente a los actores involucrados.

\section{Referencias}

[1] A. Manrubia, "El proceso productivo del videojuego: fases de producción," HICS, vol. 19, pp. 791-805, may 2014. https://doi.org/10.5209/rev_HICS.2014. v19.45178

[2] Forbes, "The World's Most Valuable Esports Companies," 2018. [Online]. Recuperado de https://www.forbes. com/sites/mikeozanian/2018/10/23/ the-worlds-most-valuable-esports-companies-1/\#29394bf36a6e. 
[3] BID, "Los videojuegos no son un juego," 2019. [Online]. Recuperado de: https:// publications.iadb.org/publications/spanish/document/Los_videojuegos_no_ son_un_juego_Los_desconocidos_\%C3\%A9xitos_de_los_estudios_de_Am\%C3\%A9rica_Latina_y_el_Caribe.pdf.

[4] (CCB), "Diagnóstico y plan de acción para la industria de animación digital y videojuegos de Bogotá," Bogotá, CCB, 2010. [Online]. Recuperado de https:// bibliotecadigital.ccb.org.co/bitstream/ handle/11520/3000/7572diagnosticoanim1. pdf? sequence=1\&isAllowed =y

[5] (CCB), "Videojuegos mueven \$792.000 millones en el mercado local," may 2017. [Online]. Recuperado de https://www.ccb.org.co/Clusters/Cluster-de-Industrias-Creativas-y-Contenidos/Noticias/2017/Mayo/ Videojuegos-mueven-792.000-millones-en-el-mercado-local

[6] P. Alonqueo Boudon and L. Rehbein, "Usuarios habituales de videojuegos: una aproximación inicial," vol. 16, no. 29, nov 2008. [Online]. Available: https://www.researchgate.net/ publication/38104941_Usuarios_habituales_de_videojuegos_una_aproximacion_inicial.

[7] M. Danieli Franco and I. F. Dueñas Silvera, "Plan de Negocio: GGaming esports Center," 2016. [Online]. Recuperado de: http://tesis.pucp.edu.pe/repositorio/handle/20.500.12404/9376 =y.

[8] A. Álvarez Estrepo and J. Barrera Gómez, "eSports: Los videojuegos como deporte emergente," 2018. [Online]. Recuperado de https://revistas.udea. edu.co/index.php/expomotricidad/article/view/336099/20791642.
[9] NewZoo, "2018 global games. Market report," 2018. [Online]. Recuperado de https://cdn2.hubspot.net/ hubfs/700740/Reports/Newzoo_2018_ Global_Games_Market_Report_Light. pdf.

[10] PROCOLOMBIA, "Unity elige a Colombia como plataforma de expansión regional," s.f. [Online]. Recuperado de https://procolombia.co/multimedia/video/unity3d-elige-colombia-como-plataforma-de-expansion-regional. 